\title{
Spectral Estimates of a Wind Fluctuation Statistic Pertaining to Wind Energy Generators
}

T. C. Kerrigan

Atmospheric Physics Section

September 1978

Prepared for the U.S. Department of Energy under Contract No. EY-76-C-06-1830

Pacific Northwest Laboratory Operated for the U.S. Department of Energy by 


\title{
NOTICE
}

This report was prepared as an account of work sponsored by the United States Government. Neither the United States nor the Department of Energy, nor any of their employees, nor any of their contractors, subcontractors, or their employees, makes any warranty, express or implied, or assumes any legal liability or responsibility for the accuracy, completeness or usefulness of any information, apparatus, product or process disclosed, or represents that its use would not infringe privately owned rights.

The views, opinions and conclusions contained in this report are those of the contractor and do not necessarily represent those of the United States Government or the United States Department of Energy.

\author{
PACIFIC NORTHWEST LABORATORY \\ operated by \\ BATTELLE \\ for the \\ UNITED STATES DEPARTMENT OF ENERGY \\ Under Contract EY-76-C-06-1830
}

\author{
Printed in the United States of America \\ Available from \\ National Technical Information Service \\ United States Department of Commerce \\ 5285 Port Royal Road \\ Springfield, Virginia 22751
}

Price: Printed Copy $\$$ -; Microfiche $\$ 3.00$

NTIS
- Pages Selling Price

$001-025 \quad \$ 400$

$026-050 \quad \$ 4.50$

$057-075 \quad \$ 5.25$

$076-100 \quad \$ 6.00$

$101-i 25 \quad \$ 6.30$

$126.750 \quad \$ 7.25$

$151-175 \quad \$ 8.00$

$176.200 \quad \$ 9.00$

201-225 \$9.25

226-250 \$9.50

251-275 $\$ \$ 10.75$

$275-300 \quad \$ 11.00$ 
SPECTRAL ESTIMATES OF A WIND FLUCTUATION STATISTIC PERTAINING TO WIND ENERGY GENERATORS

T. C. Kerrigan

Atmospheric Physics Section

September 1978

Prepared for the U.S. Department of Energy under Contract EY-75-C-06-1830

Pacific Northwest Laboratory Richland, Washington 99352 


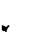




\section{$\underline{\text { SUMMARY }}$}

The object of this paper is to develop an estimate of the Erequency with which a volume average of the longitudinal component of wind velocity changes by a given amount in a given time. First, a general stochastic model for the longitudinal component of atmospheric turbulence is explicitly constructed. Second, the mathematical foundations on which this paper rests are stated. They include Rice's theorem on level crossings and a modification of the power spectrum for the longitudinal wind component to account for the volume averaging induced by a windmill rotor. Third, these mathematical results are applied to the general wind model to obtain an estimate of the frequency described above in terms of general spectral information. Fourth, attention is restricted to a stochastic wind model based on the theory of isotropic turbulence in order to obtain this estimate in terms of commonly available spectral information. And fifth, a summary and sample calculations are provided. 


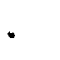


CONTENTS

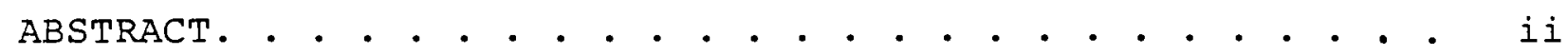
ACKNOWLEDGMENTS . . . . . . . . . . . . . . . . . . . . iv I. INTRODUCTION . . . . . . . . . . . . . . . . . . . . . . . 1 II. THE GENERAL WIND MODEL . . . . . . . . . . . . . . . . 4 III. AN ESTIMATE BASED ON THE GENERAL WIND MODEL. • • • • . 8 IV. AN ESTIMATE BASED ON AN ISOTROPIC TURBULENCE MODEL . . 11 V. SUMMARY AND SAMPLE CALCULATIONS. . . . . . . . . . . . 16 VI. CONCLUDING REMARKS • • • • • • • • • • . . . . • . . 21 REFERENCES. • . . . . . . . . . . . . . . . . . . . . 22 APPENDIX A: MATHEMATICAL FOUNDATIONS . . . . . . . . . . A-I APPENDIX B: FOURIER TRANSFORMS OF FUNCTIONS DESIGNATING AFFECTED VOLUME FOR TWO CANONICAL ROTOR GEOMETRIES • . • • . . . . . . . . . . . . $\mathrm{B}-1$ 


\section{ACKNOWLEDGMENTS}

Many thanks to $T$. J. Bander for performing the calculations presented in section $\mathrm{V}$. 


\section{INTRODUCTION}

Atmospheric turbulence is generally thought to complicate the operation and design of wind energy generators ${ }^{[1]}$. Rapid changes in the wind in the direction of the mean flow degrade power output and may threaten structural integrity. Thus, an estimate of the frequency with which this wind component changes by a given amount in a given time is regarded as important.

This paper develops such an estimate in the context of two mathematically explicit spectral models for the wind. Each model represents the longitudinal component of wind velocity as a normal stationary stochastic process which satisfies the hypotheses of the spectral theorem. This estimate then emerges as a corollary of a theorem on level crossings originally given by $S .0$. Rice ${ }^{[2]}$. He computes the expected number of times a normal scalar process crosses a level $u$ in the interval [0,T] in terms of its first several spectral moments. We derive a process from the stochastic model for the longitudinal wind component such that a level crossing for the derived process may be interpreted as a change in this wind component by an amount $\Delta$ in time $2 \tau$. Rice's result then yields the expected number of such changes in $[0, T]$ in terms of the first several spectral moments of the derived process.

Rice's result does not apply directly to this estimation problem, however. The obstacle is that most popular characterizations of the power spectral density for the longitudinal 
component of atmospheric turbulence fall off according to a 5/3-power law ${ }^{[3]}$. This asymptotic behavior implies the divergence of the second spectral moment for the derived process, which in turn implies an infinite expected number of wind events of the kind described above.

These characterizations fail to account for two significant physical effects, however. The first is viscous depletion of kinetic energy in the higher spectral frequencies [4]. Although including viscous depletion results in the convergence of the second spectral moment for the derived process, the specific numerical value of the result is sensitive to the distribution of energy through the entire range of frequencies. The second effect is the virtual volume averaging of the wind field by the presence of the windmill rotor. Including this averaging also results in the convergence of the second spectral moment for the derived process, since it effectively diminishes the role of the higher spectral frequencies. In the course of this paper, we shall modify our spectral models by including this second effect. Besides accounting for windmill geometry and providing for the convergence of the second spectral moment, this volume averaging dominates the less assessable influence of viscous depletion.

Finally, we observe the following outline of the derivation of this spectral estimate. First, we explicitly construct a general stochastic model for the longitudinal component of atmospheric turbulence. Second, we state the mathematical foundations on which this paper rests. They consist of Rice's theorem 
on level crossings, a construction of the spectrum for the derived process, and a modification of this spectrum to account for volume averaging. Third, we apply these mathematical results to the general wind model to obtain an estimate of the statistic we seek in terms of general spectral information. Fourth, we restrict attention to a stochastic model based on the theory of isotropic turbulence in order to obtain this estimate in terms of commonly available spectral information. And fifth, we provide a summary of the results which are most useful in computing this estimate and some sample calculations which serve to illustrate these results. 


\section{THE GENERAI WIND MODEL}

The object of this section is to construct a stochastic process which will serve as the general model for the wind component in the direction of the mean flow.

Let $t$ denote time and $\mathrm{x}$ denote position. We write $\mathrm{x}=\left(\mathrm{x}_{1}, \mathrm{x}_{2}, \mathrm{x}_{3}\right)$ for a general element in the three-dimensional Enclidean space $E_{3}$. We specify that $x_{1}$ correspond to the longitudinal direction (direction of the mean flow), $x_{2}$ to the lateral direction, and $x_{3}$ to the vertical direction. We take the earth's surface to be a plane normal to the vertical axis, center the coordinate system above this surface and assume that the wind speed is identically zero in the opposite half-space.

We assume that the longitudinal component of wind velocity is modeled by a stochastic process $u(t, x)$ which is stationary in $t$, homogeneous in $\left(x_{1}, x_{2}\right)$, and continuous in quadratic mean. Let $E$ denote expectation and define the mean $U$ and the covariance function $\mathrm{R}$ by

$$
U(t, x) \equiv E u(t, x),
$$

and

$$
R(t, x, y) \equiv E(u(t, x)-u(t, x))(u(t, y)-U(t, y)) .
$$

Stationarity and homogeneity imply that $\mathrm{U}$ depends on just $\mathrm{x}_{3}$, and stationarity implies that $R$ is independent of $t$. Unfortunately, the spectral theorem does not apply directly to the 
spatial analysis of the $u$-process since $R$ lacks translation invariance in $x_{3}$. We proceed to replace $u$ by an associated homogeneous process $u^{\prime}$, however, in order to take advantage of the spectral approach.

Let $k$ denote a function on $\mathrm{E}_{3}$ having the following properties:

$$
\begin{aligned}
& \text { 1) } k(x) \geq 0 \text { for all } x \text { in } E_{3} \text {, } \\
& \text { 2) } k(x)=0 \text { for }|x| \geq r \text { for some } r \text {, } \\
& \text { 3) } \int_{E_{3}} k(x) d x=1 .
\end{aligned}
$$

$k$ serves to define the region of space in which the wind has an influence on the windmill rotor. A precise specification of the actual region of influence is quite abeyond the scope of the present development. Two preliminary recommendations are offered in Appendix B, however. They correspond to shapes associated with popular rotor geometries, namely, a disk and a sphere.

Now set

$$
R^{\prime}(x) \equiv \int_{E_{3}} \frac{1}{2}[R(x+y, y)+R(-x+y, y)] k(y) d y
$$

$R^{\prime}$ is just the symmetrized volume average of the covariance function over the volume defined by $k$. Let $\left\{z_{1}, \ldots, z_{n}\right\}$ denote a set of $n$ complex numbers and $\left\{w_{1}, \ldots, w_{n}\right\}$ a set of $n$ vectors in $E_{3}$. Then 


$$
\begin{aligned}
\sum_{i, j=1}^{n} R^{\prime}\left(w_{i}-w_{j}\right) z_{i} \overline{z_{j}} \\
\quad=\frac{1}{2} \int_{E_{3}\left[\sum_{i, j=1}^{n} R\left(w_{i}+y, w_{j}+y\right) z_{i} \overline{z_{j} k\left(w_{j}+y\right)}\right] d y} \\
\quad+\frac{1}{2} \int_{E_{3}}\left[\sum_{i, j=1}^{n} R\left(w_{j}+y, w_{i}+y\right) z_{i} k\left(w_{i}+y\right) \overline{z_{j}}\right] d y \\
\geq 0
\end{aligned}
$$

since the quantities in square brackets are nonnegative. That is, $R^{\prime}$ inherits nonnegative definiteness from $R$. Now since $R^{\prime}$ is also symmetric, it is the covariance function for a unique (up to equivalence) mean-zero normal homogeneous stochastic process $u^{\prime}(x)^{[2]}$.

In the spirit of Taylor's hypothesis [5], we invest u' with a time dependence by taking

$$
u^{\prime}\left(t, x_{1}, x_{2}, x_{3}\right) \equiv u^{\prime}\left(x_{1}-u^{\prime} t, x_{2}, x_{3}\right)
$$

where

$$
U^{\prime} \equiv \int_{E_{3}} U(x) k(x) d x
$$

We shall see that accounting for time in this way is actually just a convenient mathematical device. In practice, we shall rely on a much weaker version of this sample path assertion, i.e.,

$$
E u^{\prime}(s+t, x) u^{\prime}(s, x)=E u^{\prime}(s, x+y) u^{\prime}(s, x),
$$


where

$$
y \equiv\left(-U^{\prime} t, 0,0\right)
$$

Henceforth, we shall regard $U^{\prime}+u^{\prime}(t, x)$ as the general wind model. In some sense, $u^{\prime}(t, x)$ is the normal stationaryhomogeneous process most closely associated with the longitudinal component of velocity fluctuations in the volume affecting the windmill rotor. We now proceed to the next section, where we use this model in analyzing these fluctuations. 


\section{AN ESTIMATE BASED ON THE GENERAI WIND MODEL}

The object of this section is to estimate the expected number of times $t$ in $[0, T]$ that a volume average of the longitudinal wind component changes by an amount $\Delta$ between $t-\tau$ and $t+\tau$. This estimate is based on the general wind model constructed in Section II. We may restrict attention to $u^{\prime}$ at the onset, however, since the remaining term in the model is time-independent.

We begin by recalling the definitions of the operators $D_{\tau}$ and $K$ given in Appendix $A$ :

$$
D_{\tau} \xi(t) \equiv \xi(t+\tau)-\xi(t-\tau)
$$

and

$$
\mathrm{K} \psi(\mathrm{x}) \equiv \int_{\mathrm{E}_{3}} \mathrm{k}(\mathrm{x}+\mathrm{y}) \psi(\mathrm{y}) \mathrm{dy}
$$

where we identify this function $\mathrm{k}$ with that given in section II. We next define $v(t)$ by

$$
v(t) \equiv D_{\tau} K u^{\prime}(t, 0)
$$

for each sample path in the $u^{\prime}(t, x)$-process. Clearly, the $v(t)-$ process is normal, stationary and such that $E v(t)=0$ for each real $t$. In addition, we assume sufficient conditions on $R(x, y)$ to make $v(t)$ continuous both almost surely and in quadratic mean. Thus, the results of Appendix A apply to the $v(t)$-process. 
Let $D_{\Delta, \tau}(0, T)$ denote the number of times $t$ in $[0, T]$ that $v(t)=\Delta$. Then $E\left\{D_{\Delta, \tau}(0, T)\right\}$ is just the expected number of times $t$ in $[0, T]$ that the volume average of u' associated with $k$ changes by an amount $\Delta$ between $t-\tau$ and $t+\tau$. According to the version of Rice's theorem stated in Proposition Al,

$$
E\left\{D_{\Delta, \tau}(0, T)\right\}=\frac{T}{\pi}\left(\frac{\lambda_{2}}{\lambda_{0}}\right)^{1 / 2} \exp \left(-\frac{\Delta^{2}}{2 \lambda_{0}}\right)
$$

where $\lambda_{n}$ ' denotes the $n^{\text {th }}$ spectral moment for the $v(t)$-process. Thus, obtaining the estimate we seek is now simply a matter of computing $\lambda_{0}$ ' and $\lambda_{2}{ }^{\prime}$.

Set

$$
\phi^{\prime}(k) \equiv \frac{1}{(2 \pi)^{3}} \int_{E_{3}} e^{-i k \cdot x} R^{\prime}(x) d x
$$

the spectral density for the $\mathrm{u}^{\prime}(\mathrm{x})$-process. First, Remark A2 implies that

$$
(2 \pi)^{6}|F k(k)|^{2} \phi^{\prime}(k)
$$

is the spectral density for the $\mathrm{Ku}^{\prime}(\mathrm{x})$-process. Next, remark Al and the stochastic equivalence of tine and space hypothesized in section II inply that

$$
4(2 \pi)^{6} \sin ^{2}\left(U^{\prime} \tau \kappa_{I}\right)|F k(k)|^{2} \phi^{\prime}(K)
$$


is the spectral density for the $D_{\tau} \mathrm{Ku} \mathrm{u}^{\prime}(t, \mathrm{x})$-process. Continuing to regard $t$ as a parameter, we have that

$$
4(2 \pi)^{6} \sin ^{2}\left(U^{\prime} \tau \kappa_{1}\right) \int_{E_{2}}|F k(k)|^{2} \cdot \phi^{\prime}(k) d k_{2} d \kappa_{3}
$$

is the spectral density for the $D_{\tau} K^{\prime}\left(t, x_{1}, 0,0\right)$-process. Finally, returning to the temporal point of view, we have that

$$
4(2 \pi)^{6} \frac{\sin ^{2}(\tau \lambda)}{U^{\prime}} \int_{E_{2}}\left|F k\left(\frac{-\lambda}{U^{\top}}, k_{2}, k_{3}\right)\right|^{2} \phi^{\prime}\left(\frac{-\lambda}{U^{\top}}, k_{2}, k_{3}\right) d k_{2} d k_{3}
$$

is the spectral density for the $v(t)$-process. We obtain $\lambda_{0}$ ' and $\lambda_{2}$ ' from this density according to the definition of spectral moments given in Appendix A. Thus, given $R(x, y)$, it is now a simple matter in principle to compute $E\left\{D_{\Delta, \tau}(0, T)\right\}$. 
IV. AN ESTIMATE BASED ON AN

ISOTROPIC TURBULENCE MODEL

Even if $\phi^{\prime}$ were generally available, estimating $\lambda_{0}$ ' and $\lambda_{2}$ ' would still be encumbered by the double integration in the representation of the spectral density for the $v(t)$-process. We now turn to a stochastic model for the wind which is based on the theory of isotropic turbulence. Although this model fails to account for statistical inhomogeneities in the flow field, it compensates by furnishing tractable estimates for $\lambda_{0}$ ' and $\lambda_{2}$ ' in terms of more readily available spectral information.

At the onset, we model the longitudinal component of wind velocity by a normal, stationary and homogeneous stochastic process $u(t, x)$. We set $U \equiv E u(t, x)$ and assume that the version of Taylor's hypothesis described in section II holds. We further assume that the sample paths are continuous both almost surely and in quadratic mean. Now define the $w(t)$-process by taking

$$
w(t) \equiv D_{\tau} K u(t, 0)
$$

for each sample path $u(t, x)$. Let $D_{\Delta, \tau}(0, T)$ denote the number of times $t$ in $[0, T]$ that $w(t)=\Delta$. As in Section III,

$$
E\left\{D_{\Delta, \tau}(0, T)\right\}=\frac{T}{\pi}\left(\frac{\lambda_{2}^{\prime}}{\lambda_{0}^{\top}}\right)^{1 / 2} \exp \left(-\frac{\Delta^{2}}{2 \lambda_{0}}\right)
$$

where $\lambda_{n}$ ' denotes the $n^{\text {th }}$ spectral moment for the $w(t)$-process. 
We proceed to compute estimates for $\lambda_{0}$ ' and $\lambda_{2}$ ' based on the theory of isotropic turbulence. This section essentially repeats much of section III. As in section III, we find it convenient to suppress the explicit dependence of $u$ on time during the computation of the spectral density for the $w(t)$-process.

Let $\mathrm{u}_{1}, \mathrm{u}_{2}$ and $\mathrm{u}_{3}$ denote the longitudinal, lateral and vertical components of the turbulence model. (We have identified $\mathrm{u}_{1}$ and $\left.\mathrm{u}-\mathrm{U}\right)$. Assume that this vector process is isotropic. Define the covariance tensor by

$$
R_{i j}(x) \equiv E u_{i}(x+y) u_{j}(y)
$$

and the spectral density tensor by

$$
\Phi_{i j}(k) \equiv \frac{1}{(2 \pi)^{3}} \int_{E_{3}} e^{-i k \cdot x} R_{i j}(x) d x
$$

Let $\phi$ denote the spectral density for the $u_{1}\left(x_{1}, 0,0\right)$-process:

$$
\phi(\lambda)=\frac{1}{2 \pi} \int_{-\infty}^{\infty} e^{-i \lambda x_{1}} R_{11}\left(x_{1}, 0,0\right) d x_{1}
$$

According to the theory of isotropic turbulence $[5], \Phi_{i j}$ is given by

$$
\Phi_{i j}(k)=\frac{E(|k|)}{4 \pi|k|^{4}}\left(|k|^{2} \delta_{i j}-k_{i} k_{j}\right)
$$


where

$$
E(\lambda) \equiv \lambda^{3} \frac{d}{d \lambda}\left[\frac{1}{\lambda} \frac{d \phi}{d \lambda}(\lambda)\right]
$$

As in Section III, the spectral density for the $w(t)$-process is given by

$$
\begin{aligned}
4(2 \pi)^{6} & \frac{\sin ^{2}(\tau \lambda)}{U} \int_{E_{2}}\left|F k\left(\frac{-\lambda}{U}, k_{2}, \kappa_{3}\right)\right|^{2} \Phi_{11}\left(\frac{-\lambda}{U}, k_{2}, k_{3}\right) d k_{2} d k_{3} \\
= & 2(2 \pi)^{5} \frac{\sin ^{2}(\tau \lambda)}{U} \int_{E_{2}}\left|F k\left(\frac{-\lambda}{U}, \kappa_{2}, k_{3}\right)\right|^{2}\left(\kappa_{2}{ }^{2}+\kappa_{3}^{2}\right) \\
& {\left.\left[\left(\frac{1}{\sigma} \frac{d}{d \sigma}\right)^{2} \phi(\sigma)\right]\right|_{\sigma=\sqrt{\left(\frac{\lambda}{U}\right)^{2}+\kappa_{2}{ }^{2}+\kappa_{3}{ }^{2}}} d k_{2} d k_{3} . }
\end{aligned}
$$

It is now a simple matter in principle to compute $\lambda_{0}{ }^{\prime}$ and $\lambda_{2}{ }^{\prime}$, from which we obtain the estimate $E\left\{D_{\Delta, \tau}(O, T)\right\}$ in the context of isotropic turbulence.

The assumption of isotropy allows us to make a considerable simplification at this point. We refer to Appendix $B$ for the computation of $F k$ for $k$ corresponding to the geometry of two basic windmill rotor configurations--the disk and the sphere. In both cases $|F k(k)|^{2}$ assumes the form $G\left(k_{1}, k_{2}{ }^{2}+k_{3}{ }^{2}\right)$. We substitute this form and proceed to compute simplified expressions for $\lambda_{0}$ ' and $\lambda_{2}{ }^{\prime}$ : 


$$
\begin{gathered}
\left.\int_{E_{2}} G\left(\frac{-\lambda}{\mathrm{U}}, \kappa_{2}{ }^{2}+\kappa_{3}{ }^{2}\right)\left({\kappa_{2}}^{2}+\kappa_{3}{ }^{2}\right)\left[\left(\frac{1}{\sigma} \frac{d}{d \sigma}\right)^{2} \phi(\sigma)\right]\right|_{\sigma=\sqrt{\left(\frac{\lambda}{U}\right)^{2}+\kappa_{2}{ }^{2}+\kappa_{3}{ }^{2}}} d \kappa_{2} d \kappa_{3} \\
\quad=\left.\int_{0}^{2 \pi} d \theta \int_{0}^{\infty} \mathrm{dr} r^{3} G\left(\frac{-\lambda}{U}, r^{2}\right)\left[\left(\frac{1}{\sigma} \frac{d}{d \sigma}\right)^{2} \phi(\sigma)\right]\right|_{\sigma=\sqrt{\left(\frac{\lambda}{U}\right)^{2}+r^{2}}} \\
=2 \pi \int_{\frac{\lambda}{U}}^{\infty}\left(\sigma^{2}-\left(\frac{\lambda}{U}\right)^{2}\right) G\left(\frac{-\lambda}{U}, \sigma^{2}-\left(\frac{\lambda}{U}\right)^{2}\right) \frac{d}{d \sigma}\left(\frac{1}{\sigma} \frac{d \phi}{d \sigma}(\sigma)\right) d \sigma .
\end{gathered}
$$

Thus,

$$
\begin{aligned}
\lambda_{0}^{\prime}= & 4(2 \pi)^{6} \int_{0}^{\infty} \mathrm{d} \lambda \frac{\sin ^{2}(\tau \lambda)}{U} \int_{\frac{\lambda}{U}}^{\infty} d \sigma\left(\sigma^{2}-\left(\frac{\lambda}{U}\right)^{2}\right) G\left(\frac{-\lambda}{U}, \sigma^{2}-\left(\frac{\lambda}{U}\right)^{2}\right) \\
& \frac{d}{d \sigma}\left(\frac{1}{\sigma} \frac{d \phi}{d \sigma}(\sigma)\right) \\
= & 4(2 \pi)^{6} \int_{0}^{\infty} d \sigma \frac{d}{d \sigma}\left(\frac{1}{\sigma} \frac{d \phi}{d \sigma}(\sigma)\right) \int_{0}^{\sigma} d \lambda \sin ^{2}(U \tau \lambda)\left(\sigma^{2}-\lambda^{2}\right) G\left(-\lambda, \sigma^{2}-\lambda^{2}\right) \\
= & -4(2 \pi)^{6} \int_{0}^{\infty} d \sigma \frac{d \phi}{d \sigma}(\sigma) \frac{1}{\sigma} \int_{0}^{\sigma} d \lambda \sin ^{2}(U \tau \lambda) \frac{d}{d \sigma}\left[\left(\sigma^{2}-\lambda^{2}\right)\right. \\
& \left.G\left(-\lambda, \sigma^{2}-\lambda^{2}\right)\right]
\end{aligned}
$$




$$
\begin{aligned}
= & 4(2 \pi)^{6} \int_{0}^{\infty} d \sigma \frac{d \phi}{d \sigma}(\sigma) \frac{1}{\sigma} \int_{0}^{\sigma} d \lambda \frac{1}{2}(1-\cos (2 \mathrm{U} \tau \lambda)) \\
& \left.\frac{d}{d \lambda}\left[\left(\sigma^{2}-\lambda^{2}\right) \mathrm{G}\left(-\delta, \sigma^{2}-\lambda^{2}\right)\right]\right|_{\delta=\lambda} \\
= & -4 \int_{0}^{\infty} \mathrm{d} \sigma \frac{\mathrm{d} \phi}{\mathrm{d} \sigma}(\sigma) \frac{1}{\sigma} \int_{0}^{\sigma} \mathrm{d} \lambda\left(\sigma^{2}-\lambda^{2}\right) \mathrm{U} \tau \sin (2 \mathrm{U} \tau \lambda) \\
& (2 \pi)^{6} \mathrm{G}\left(-\lambda, \sigma^{2}-\lambda^{2}\right) .
\end{aligned}
$$

Similarly,

$$
\begin{aligned}
\lambda_{2}{ }^{\prime}= & -4 \int_{0}^{\infty} d \sigma \frac{d \phi}{d \sigma}(\sigma) \frac{1}{\sigma} \int_{0}^{\sigma} d \lambda\left(\sigma^{2}-\lambda^{2}\right)\left[2 U^{2} \lambda \sin ^{2}(U \tau \lambda)\right. \\
& \left.+U^{3} \tau \lambda^{2} \sin (2 U \tau \lambda)\right](2 \pi)^{6} G\left(-\lambda, \sigma^{2}-\lambda^{2}\right)
\end{aligned}
$$

Therefore, given $\phi, \tau$ and $G$, it is now a simple matter to compute $E\left\{D_{\Delta, \tau}(0, T)\right\}$ using these expressions for $\lambda_{0}$ ' and $\lambda_{2}$ ', and the version of Rice's theorem stated earlier in this section. 


\section{SUMMARY AND SAMPLE CALCULATIONS}

This section consists of a statement of the most useful results developed in this paper and some sample computations which serve to illustrate these results. Recall that $E\left\{D_{\Delta, \tau}(0, T)\right\}$ denotes the expected number of times $t$ in $[0, T]$ that a volume average of the longitudinal wind component changes by an amount $\Delta$ between $t-\tau$ and $t+\tau$. An estimate of this expected number based on Rice's theorem is given by

$$
E\left\{D_{\Delta, T}(0, T)\right\}=\frac{T}{\pi}\left(\frac{\lambda 2^{\prime}}{\lambda_{0}}\right)^{1 / 2} \exp \left(-\frac{\Delta^{2}}{2 \lambda_{0}^{\top}}\right)
$$

whẹre $\lambda_{0}$ ' and $\lambda_{2}$ ' denote spectral moments of a modified power spectrum for atmospheric turbulence. Assuming an isotropic turbulence model, we have that

$\lambda_{0}^{\prime}=-4 \int_{0}^{\infty} d \sigma \frac{\mathrm{d} \phi}{\mathrm{d} \sigma}(\sigma) \frac{1}{\sigma} \int_{0}^{\sigma} \mathrm{d} \lambda\left(\sigma^{2}-\lambda^{2}\right) \mathrm{U} \tau \sin (2 \mathrm{U} \tau \lambda)(2 \pi)^{6} \mathrm{G}\left(-\lambda, \sigma^{2}-\lambda^{2}\right)$

and

$$
\begin{aligned}
\lambda_{2}{ }^{\prime}= & -4 \int_{0}^{\infty} d \sigma \frac{d \phi}{d \sigma}(\sigma) \frac{1}{\sigma} \int_{0}^{\sigma} d \lambda\left(\sigma^{2}-\lambda^{2}\right)\left[2 U^{2} \lambda \sin ^{2}(U \tau \lambda)\right. \\
& \left.+U^{3} \tau \lambda^{2} \sin (2 U \tau \lambda)\right](2 \pi)^{6} G\left(-\lambda, \sigma^{2}-\lambda^{2}\right),
\end{aligned}
$$

where $\phi$ denotes the (whole-line) power spectral density for the longitudinal component of atmospheric turbulence, $U$ denotes the 
mean wind speed and $G$ is a function associated with windmill geometry. By Appendix B,

$$
(2 \pi)^{\sigma} G\left(-\lambda, \sigma^{2}-\lambda^{2}\right)=4\left[\frac{\sin a \lambda}{a \lambda} \frac{J_{I}\left(b \sqrt{\sigma^{2}-\lambda^{2}}\right)}{b \sqrt{\sigma^{2}-\lambda^{2}}}\right]^{2}
$$

for a disk of thickness $2 a$ and radius $b$, where the a-axis is oriented parallel to the mean wind velocity and $J_{1}$ denotes the first order Bessel function, and

$$
(2 \pi)^{6} G\left(-\lambda, \sigma^{2}-\lambda^{2}\right)=9\left[\frac{j_{l}(c \lambda)}{c \lambda}\right]^{2}
$$

for a sphere of radius $c$, where $j_{l}$ denotes the first order spherical Bessel function.

We have performed several calculations of $E\left\{D_{\Delta, \tau}(0, T)\right\}$ based on the theory summarized in this section. We confine attention to the geometry of a disk and to the Von Karman spectrum, which is given by

$$
\phi(\sigma) \equiv \frac{L\left(k_{v} U\right)^{2}}{\pi}\left[I+(1.339 L \sigma)^{2}\right]^{-5 / 6},
$$

where $L$ denotes turbulent length scale and $k_{v}$ denotes turbulence intensity [3]. For definiteness, we take

$$
\begin{aligned}
& \mathrm{a}=0.15 \mathrm{~m}, \\
& \mathrm{~b}=4.5 \mathrm{~m}, \\
& \mathrm{k}_{\mathrm{v}}=0.2 \text { and } 0.3,
\end{aligned}
$$




$$
\begin{aligned}
& \mathrm{L}=60.0 \mathrm{~m}, \\
& \mathrm{~T}=1.0 \text { hours, } \\
& \mathrm{U}=6.7 \mathrm{~m} / \mathrm{sec}(15.0 \mathrm{mi} / \mathrm{hr}) .
\end{aligned}
$$

The results of these calculations are given in Tables 1 and 2. Table 1 presents values of $E\left\{D_{\Delta, \tau}(0, T)\right\}$ for various values of $\Delta$ and $\tau$, and $k_{v}=0.2$; Table 2 presents values of $E\left\{D_{\Delta, \tau}(O, T)\right\}$ for various values of $\Delta$ and $\tau$, and $k_{v}=0.3$. 

Table 1. $E\left\{D_{\Delta, \tau}(0, T)\right\}$ for various values
of $\Delta$ and $\tau$, and $k_{V}=0.2$

\begin{tabular}{|c|c|c|c|c|c|}
\hline$\Delta$ & 0.125 & 0.25 & 0.5 & 1.0 & 2.0 \\
\hline 2.0 & $1.3 \times 10^{-53}$ & $1.0 \times 10^{-13}$ & $4.2 \times 10^{-3}$ & 5.2 & $5.3 \times 10^{1}$ \\
4.0 & $\sim 0$ & $4.4 \times 10^{-63}$ & $3.1 \times 10^{-20}$ & $2.4 \times 10^{-7}$ & $6.9 \times 10^{-3}$ \\
6.0 & $\sim 0$ & $\sim 0$ & $8.9 \times 10^{-49}$ & $1.4 \times 10^{-19}$ & $2.3 \times 10^{-9}$ \\
8.0 & $\sim 0$ & $\sim 0$ & $9.8 \times 10^{-89}$ & $1.1 \times 10^{-36}$ & $2.0 \times 10^{-18}$ \\
10.0 & $\sim 0$ & $\sim 0$ & $\sim 0$ & $1.0 \times 10^{-58}$ & $4.3 \times 10^{-30}$ \\
\hline
\end{tabular}




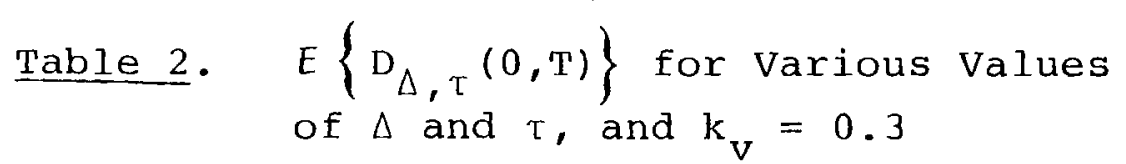

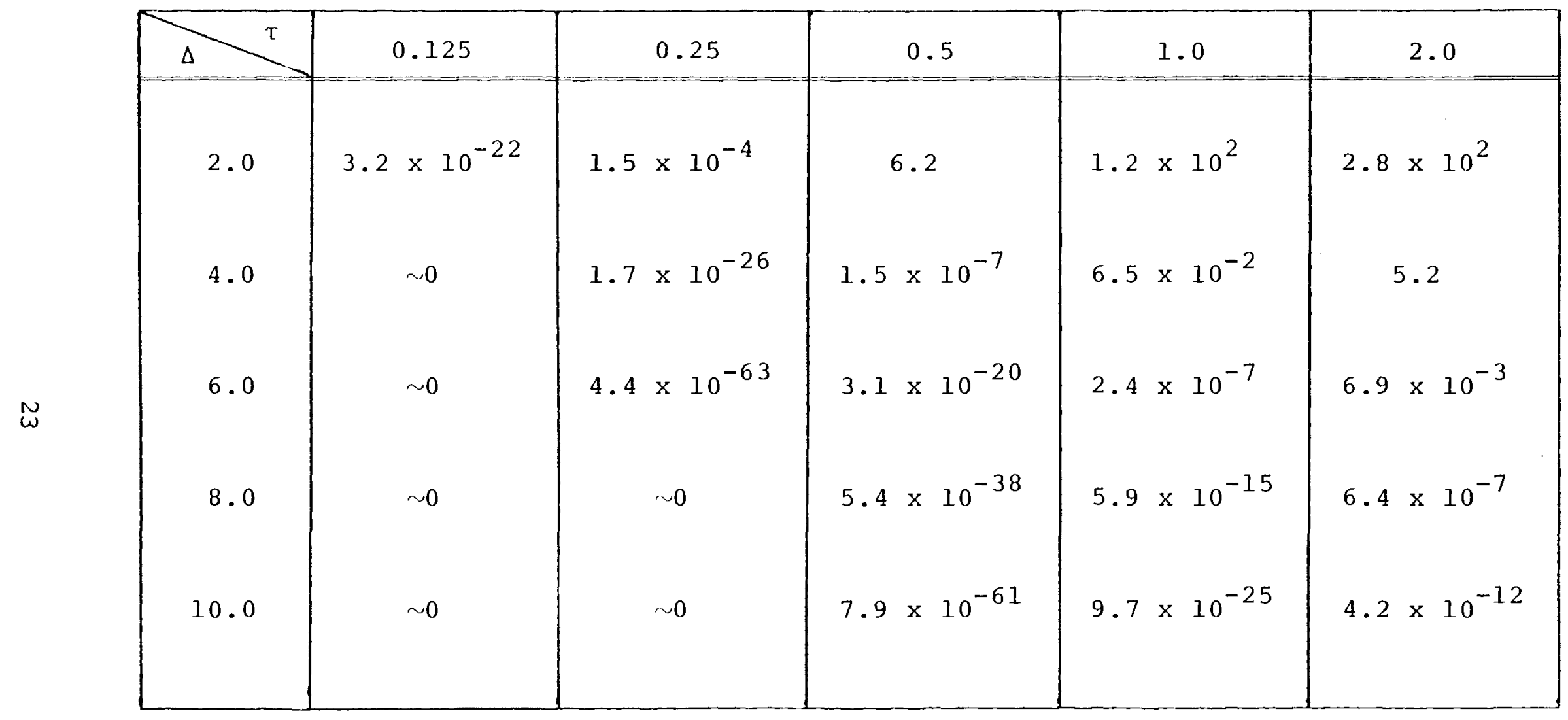




\section{CONCLUDING REMARKS}

The limits of validity for these estimates are presently unknown. Homogeneity is evidently necessary for the application of Rice's theorem. A paper examining the Gaussian assumption should be out shortly. A Battelle report comparing this estimation method with others should also be out shortly. The experimental measurements presented in it will hopefully shed some light on this issue.

Nevertheless, the estimates represented by $E\left\{D_{\Delta, \tau}(0, T)\right\}$ are possibly the best presently available. Any such estimates ultimately rest on the theory of sample path properties for stationary stochastic processes. Better estimates would correspond to deeper mathematical theorems. For example, an important generalization might be to estimate the expected number of times $t$ in $[0, T]$ that the $v$ or $w$-process changes from $a$ to $b$ between $t-\tau$ and $t+\tau$. The corresponding mathematical problem is unsolved, however.

Finally, we are fortunate in that the choices of $\mathrm{k}$ corresponding to a disk and a sphere lead to considerable mathematical simplification. We obtain this same simplification for choices of $k$ corresponding to an annular solid and a spherical shell. In fact, $F_{k}$ for these cases can be obtained from the expressions given in Appendix $B$ with little additional effort. 


\section{REFERENCES}

[1] Elderkin, C. E., J. V. Ramsdell and G. P. Tennyson: "Meeting Review: Wind Characteristics Workshop, 2-4 June 1976, Boston, MA, "Bulletin American Meteorological Society, 58:1, pp 45-51, January 1977 .

[2] Cramer, Harald and M. R. Leadbetter: Stationary and Related Stochastic Processes. Sample Function Properties and Their Applications. John wiley \& Sons, Inc. 348 pp. 1967.

[3] Daniels, Glenn E.,(ed.): Terrestrial Environment (climatic) Criteria Guidelines for use in Aerospace Vehicle Development, 1973 Revision. NASA-TM-X-64757, George C. Marshall Space Flight Center, A $\bar{L}$. Page 5.3.13, July 5, 1973.

[4] Landau, L. D. and E. M. Lifshitz: Fluid Mechanics. Pergamon Press, 536 pp. 1959.

[5] Batchelor, G. K.: The Theory of Homogeneous Turbulence. Cambridge at the University Press. 197 pp. 1970.

[6] Rudin, Walter: Principles of Mathematical Analysis. McGraw-Hill Book Company. 270 pp. 1964.

[7] Doob, J. L.: Stochastic Processes. John Wiley \& Sons, Inc., NY, 654 pp. 1953.

[8] Bell, W. W.: Special Functions for Scientists and Engineers. D. Van Nostrand Company, Ltd., London. 247 pp. 1968 . 
APPENDIX A

MATHEMATICAL FOUNDATIONS 
This section consists of a precise statement of Rice's theorem and two remarks about linear operators on spaces of sample paths. It forms the matematical basis for calculating the expectation described in the introduction. A detailed exposition of these topics may be found in [2].

First, let $\xi(t)$ denote a normal stationary stochastic process and $E$ denote expectation. We specify that $\xi(t)$ be real valued, continuous in quadratic mean and such that $E \xi(t)=0$ for all real valued $t$. Thus, the spectral theorem holds. Let $\zeta(\lambda)$ denote the orthogonal process dual to $\xi(t)$ and $d F(\lambda)$ its spectral measure, and define spectral moments $\lambda_{n}^{\cdot}$ by

$$
\lambda_{n} \equiv \int_{-\infty}^{\infty} \lambda^{n} d F(\lambda), \quad(n \geq 0) .
$$

Now let $C_{u}(0, T)$ denote the number of times $\xi(t)$ crosses the level $u$ in the interval $[0, T]$. Although the finiteness of $\lambda_{2}$ guarantees that $\xi(t)$ is equivalent to a process with continuous sample paths, we assume that $\xi(t)$ has continuous sample paths almost surely in any case. Then

Theorem Al (Rice):

$$
E\left\{C_{u}(0, T)\right\}=\frac{T}{\pi}\left(\frac{\lambda_{2}}{\lambda_{0}}\right)^{I / 2} \exp \left(-\frac{u^{2}}{2 \lambda_{0}}\right) \text {. }
$$

The case $\lambda_{2}=\infty$ is included. 
We now state the modified version of this theorem which applies to our situation. Let $\mathrm{N}_{\mathrm{u}}(0, \mathrm{~T})$ denote the number of times that $\xi(t)=u$ in $[0, T]$. Then

Proposition Al:

$$
E\left\{\mathrm{~N}_{\mathrm{u}}(0, \mathrm{~T})\right\}=E\left\{\mathrm{c}_{\mathrm{u}}(0, \mathrm{~T})\right\}
$$

The case $\lambda_{2}=\infty$ is included.

Next, define the operator $D_{\tau}$ on the sample path $\xi(t)$ by

$$
D_{\tau} \xi(t) \equiv \xi(t+\tau)-\xi(t-\tau)
$$

The $D_{\tau} \xi(t)$-process is clearly stationary, continuous in quadratic mean and such that $E D_{\tau} \xi(t)=0$ for all real valued $t$. As above, the spectral theorem holds. In particular, $D_{\tau} \xi(t)$ enjoys the following representation:

$$
\begin{aligned}
D_{\tau} \xi(t) & =\int_{-\infty}^{\infty} e^{i(t+\tau) \lambda} d \zeta(\lambda)-\int_{-\infty}^{\infty} e^{i(t-\tau) \lambda} d \zeta(\lambda) \\
& =\int_{-\infty}^{\infty} 2 i \sin (\tau \lambda) e^{i t \lambda} d \zeta(\lambda)
\end{aligned}
$$

Therefore,

Remark Al:

The spectral measure for the $D_{\tau} \xi(t)$-process is given by

$$
E|2 i \sin (\tau \lambda) d \zeta(\lambda)|^{2}=4 \sin ^{2}(\tau \lambda) d F(\lambda)
$$


Similarly, let $\psi(x)$ denote a homogeneous stochastic process such that the parameter $\mathrm{x}$ takes values in the $\mathrm{n}$-dimensional Enclidean space $E_{n}$. We specify that $\psi(x)$ be real-valued, continuous in quadratic mean and such that $E \psi(\mathrm{x})=0$ for all $\mathrm{x}$ in $E_{n}$. Then an $n$-dimensional version of the spectral theorem holds. Let $\eta(K)$ denote the orthogonal process dual to $\psi(x)$ and $d \Phi(K)$ its spectral measure.

Further, let $k$ denote an integrable function with compact support in $E_{n}[6]$, and assume that the $\psi(x)$-process has continuous sample paths almost surely. Then the operator $\mathrm{K}$ defined by

$$
\mathrm{K} \psi(\mathrm{x}) \equiv \int_{\mathrm{E}_{\mathrm{n}}} \mathrm{k}(\mathrm{x}+\mathrm{y}) \psi(\mathrm{y}) \mathrm{dy}
$$

is well-defined almost surely. In addition,

$$
\begin{aligned}
K \psi(x) & =\int_{E_{n}} k(x+y)\left(\int_{E_{n}} e^{i y \cdot k} d \eta(k)\right) d y \\
& =\int_{E_{n}}\left(\int_{E_{n}} k(x+y) e^{i(x+y) \cdot k} d y\right) e^{-i x \cdot k} d \eta(k) \\
& =\int_{E_{n}}(2 \pi)^{n} \frac{1}{\left(\frac{1 \pi)^{n}}{(2 \pi} \int_{n} e^{-i k \cdot y} k(y) d y\right)} e^{-i x \cdot k} d n(k) \\
& =\int_{E_{n}}(2 \pi)^{n} \frac{(F k)(k)}{(F x \cdot k} d \eta(k),
\end{aligned}
$$


where $F k$ denotes the Fourier transform of $k$. Note that the integrability of $\mathrm{k}$ legitimizes interchanging the order of integration ${ }^{[7]}$. Therefore,

Remark A2:

The spectral measure for the $K \psi(\mathrm{x})$-process is given by

$$
E\left|(2 \pi)^{\mathrm{n}} F \mathrm{k}(k) \mathrm{dn}(k)\right|^{2}=(2 \pi)^{2 \mathrm{n}}|\mathrm{Fk}(k)|^{2} \mathrm{~d} \Phi(k) \text {. }
$$


APPENDIX B

FOURIER TRANSFORMS OF FUNCTIONS DESIGNATING

AFFECTED VOLUME FOR TWO CANONICAL ROTOR GEOMETRIES 
The object of this section is to compute $F k$, the Fourier transform of the function specifying affected volume, for two choices of $k$.

The first choice for $k$ corresponds to a disk of thickness $2 \mathrm{a}$ and radius $\mathrm{b}$. Set

$$
\left.k_{d}(x) \equiv \frac{1}{2 \pi a b^{2}} x_{-a, a}\right]^{\left(x_{1}\right) x}\left[0, b^{2}\right]^{\left(x_{2}{ }^{2}+x_{3}{ }^{2}\right)},
$$

where

$$
x_{[\alpha, \beta]}(\gamma) \equiv\left\{\begin{array}{l}
1 \text { in case } \alpha \leq \gamma \leq \beta \\
0 \text { otherwise. }
\end{array}\right.
$$

The orientation of the disk relative to the mean wind velocity should be clear from this representation. Let $J_{n}$ denote the Bessel function of order $n^{[8]}$. Then

$$
\begin{aligned}
F k_{d}(k)= & \frac{1}{2 \pi a b^{2}} \frac{1}{2 \pi} \int_{-a}^{a} d x_{1} e^{-i k_{1} x_{1}} \frac{1}{(2 \pi)^{2}} \int_{E_{2}} d x_{2} d x_{3} e^{-i\left(k_{2} x_{2}+k_{3} x_{3}\right)} \\
& x_{\left.0, b^{2}\right]}\left(x_{2}^{2}+x_{3}^{2}\right) \\
= & \frac{1}{2 \pi a b^{2}} \frac{1}{2 \pi} \int_{-a}^{a} d x_{1} \frac{1}{k_{1}} \frac{d}{d x_{1}} \sin k_{1} x_{1} \frac{1}{(2 \pi)^{2}} \int_{0}^{b} d r r \int_{0}^{2 \pi} d \theta \\
& e^{-i \sqrt{k_{2}{ }^{2}+k_{3}^{2}} r \cos \theta}
\end{aligned}
$$




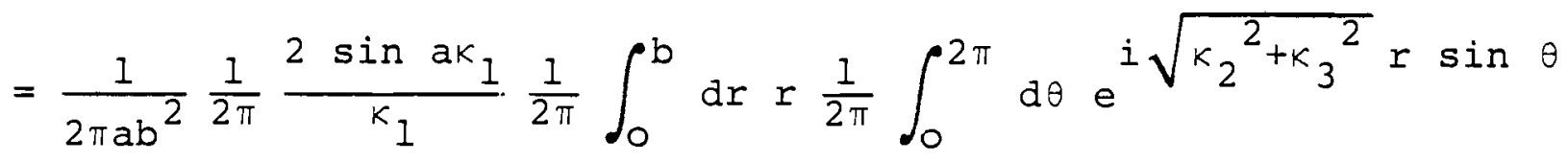

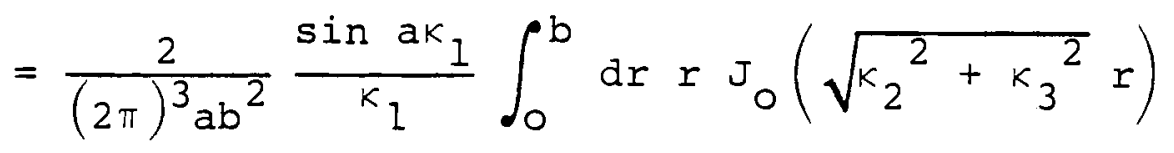

$$
\begin{aligned}
& =\frac{2}{(2 \pi)^{3} a b^{2}} \frac{\sin a \kappa_{1}}{\kappa_{1}\left(\kappa_{2}{ }^{2}+\kappa_{3}{ }^{2}\right)} \int_{0}^{b \sqrt{\kappa_{2}{ }^{2}+\kappa_{3}{ }^{2}}} d r \frac{d}{d r}\left(r J_{1}(r)\right) \\
& =\frac{2}{(2 \pi)^{3}} \frac{\sin a \kappa_{1}}{a \kappa_{1}} \frac{J_{1}\left(b \sqrt{\kappa_{2}^{2}+\kappa_{3}^{2}}\right)}{b \sqrt{\kappa_{2}{ }^{2}+\kappa_{3}^{2}}} .
\end{aligned}
$$

That is,

Proposition Bl:

$$
F k_{d}(k)=\frac{2}{(2 \pi)^{3}} \frac{\sin a k_{1}}{a k_{1}} \frac{J_{1}\left(b \sqrt{\kappa_{2}^{2}+k_{3}^{2}}\right)}{b \sqrt{k_{2}^{2}+k_{3}^{2}}}
$$

The second choice for $\mathrm{k}$ corresponds to a sphere of radius $c$. Set

$$
k_{s}(x) \equiv \frac{3}{4 \pi c^{3}} x_{[0, c]}(|x|),
$$

and let $j_{n}$ denote the spherical Bessel function of order $n$. Then

$$
F k_{S}(k)=\frac{3}{4 \pi c^{3}} \frac{1}{(2 \pi)^{3}} \int_{0}^{c} d r r^{2} \int_{S} 2 d n \cos (\alpha \hat{\kappa} \cdot n)
$$


where $\alpha \equiv r|k|, \hat{k} \equiv k /|k|$ and $s^{2}$ denotes the subset of vectors in $E_{3}$ with unit magnitude. $\int_{S^{2}} a n \cos (\alpha \hat{k} \cdot \eta)$ is clearly invariant under rotations and so is constant with respect to the argument $\hat{k}$. Thus, we may take $\hat{k} \equiv(0,0,1)$ for convenience. Coordinatizing $s^{2}$ by taking $\eta \equiv(\sin \theta \cos \phi, \sin \theta \sin \phi, \cos \theta)$, we have that

$$
\begin{aligned}
\int_{S^{2}} d n \cos (\alpha \hat{k} \cdot n) & =\int_{0}^{\pi} d \theta \int_{0}^{2 \pi} d \phi \sin \theta \cos (\alpha \cos \theta) \\
& =-\frac{2 \pi}{\alpha} \int_{0}^{\pi} d \theta \frac{d}{d \theta}(\sin (\alpha \cos \theta)) \\
& =\frac{4 \pi \sin \alpha}{\alpha} .
\end{aligned}
$$

Thus,

$$
\begin{aligned}
F k_{S}(k) & =\frac{1}{(2 \pi)^{3}} \frac{3}{4 \pi c^{3}} \int_{0}^{c} d r r^{2} 4 \pi \frac{\sin (|k| r)}{|k| r} \\
& =\frac{1}{(2 \pi)^{3}} \frac{3}{(c|k|)^{3}} \int_{0}^{c|k|} d r r \sin r \\
& =\frac{1}{(2 \pi)^{3}} \frac{3}{(c|k|)^{3}} \int_{0}^{c|k|} d r \frac{d}{d r}\left(r^{2} j_{1}(r)\right) \\
& =\frac{3}{(2 \pi)^{3}} \frac{j_{1}(c|k|)}{c|k|} .
\end{aligned}
$$

That is,

Proposition B2:

$$
F_{\mathrm{S}}(k)=\frac{3}{(2 \pi)^{3}} \frac{j_{1}(c|k|)}{c|k|} .
$$




\section{DISTRIBUTION}

No. of

Copies

OFFSITE
A. A. Churm

DOE Chicago Patent Group

9800 South Cass Avenue

Argonne, IL 60439

G. P. Tennyson

Department of Energy

600 E Street, N.W.

Washington, DC 20545

C. I. Aspliden

Department of Energy

600 E Street, N.W.

Washington, DC 20545

27 DOE Technical Information Center

10 T. C. Kerrigan

Mathematics Department

Drexel University

Philadelphia, PA 19104

ONSITE

2

DOE Richland Operations Office

G. L. Liffick

H. E. Ransom

30 Pacific Northwest Laboratory

W. C. Cliff

J. R. Connell

R. L. Conley

J. C. Doran

R. L. Drake

C. E. Elderkin (5)

D. L. Elliott

M. M. Orgill

P. R. Partch

W. T. Pennell

E. H. Phinney

J. V. Ramsdell

D. S. Renne

C. L. Simpson

L. L. Wendell (5)

Technical Information (5)

Publishing Coordination 